\title{
Cascadability Assessment of a 2R Regenerator Based on a Saturable Absorber and a Semiconductor Optical Amplifier in a Path Switchable Recirculating Loop
}

\author{
M. Gay, L. Bramerie, D. Massoubre, A. O’Hare, A. Shen, J. L. Oudar, and J. C. Simon
}

\begin{abstract}
We assess a new $2 \mathrm{R}$ regenerator based on a microcavity saturable absorber and a semiconductor optical amplifier. Cascadability is demonstrated and the impact of regeneration span is studied in a 10-Gb/s two-path recirculating loop. A wavelength study demonstrates the tunability of the device over $13 \mathbf{~ n m}$.
\end{abstract}

Index Terms-2R regeneration, optical communication, semiconductor optical amplifier (SOA), vertical microcavity saturable absorber (SA).

\section{INTRODUCTION}

A SATURABLE absorber (SA) based on a vertical microcavity is of great interest for $2 \mathrm{R}$ regeneration especially because of the possibility it offers for simultaneous regeneration of several wavelength-division-multiplexing (WDM) channels on the same chip by spatial demultiplexing [1], [2]. While an SA is efficient for noise reduction and extinction ratio enhancement at bit rates as high as $160 \mathrm{~Gb} / \mathrm{s}$ [3], complete $2 \mathrm{R}$ regeneration with limitation on the "mark" fluctuations cannot be realized without using another nonlinear function. One technique consists of spectral broadening by nonlinear propagation in a fiber and narrowband optical filtering to limit amplitude fluctuations on the marks [2]. While this technique is fairly efficient, it leads to quite a bulky device as it requires one fiber per channel, and the required power input to fiber to ensure nonlinear effects is high (typically $15 \mathrm{dBm}$ per channel).

In this letter, we investigate an alternative architecture to limit the "mark" fluctuations based on a semiconductor optical amplifier (SOA). An SA has already been used with an SOA [4] but the aim of that experiment was different since the SA was used simply to limit the amplified spontaneous emission (ASE) produced by the in-line amplifier SOA.

In our case, the impact of a $2 \mathrm{R}$ regenerator, based on an SA in a vertical microcavity cascaded with an SOA, is studied experimentally, in a transmission link. This device could signifi-

Manuscript received November 9, 2005; revised February 23, 2006. This work has been carried out in the framework of the national project ASTERIX supported by "Ministère des Finances et de l'Industrie," by Region Bretagne, and by the European Union (FEDER).

M. Gay, L. Bramerie, and J. C. Simon are with FOTON, CNRS and Université de Rennes1/ENSSAT, Lannion 22305, France (e-mail: gay@enssat.fr).

D. Massoubre and J. L. Oudar are with LPN-CNRS, F91460 Marcoussis, France.

A. O'Hare is with the School of Physics, Dublin Institute of Technology, Dublin, Ireland.

A. Shen is with the Alcatel-Thales III-V Laboratory, F91767, Palaiseau Cedex, France.

Digital Object Identifier 10.1109/LPT.2006.875324

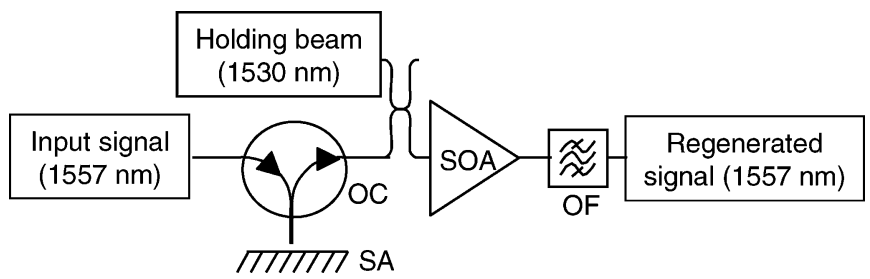

Fig. 1. All-optical regenerator architecture.

cantly increase the performance and the margin of a terrestrial link. For the first time, to our knowledge, the impact on system performance of both the regeneration span and the signal wavelength, is studied where regenerators are cascaded.

\section{REGENERATOR ARCHITECTURE}

Fig. 1 shows the regenerator architecture. The signal to be regenerated is injected onto the SA microcavity through an optical circulator. The SA is a three-quantum-well structure (InGaAs-InP) in a resonant microcavity (Ag mirror as a back mirror, Bragg reflector $\left(\mathrm{SiO}_{2}-\mathrm{TiO}_{2}\right)$ as a front mirror) both to decrease the switching threshold and to increase the switching amplitude. The cavity structure, fabricated by partners of the national research program ASTERIX, is described in more detail in [5]. The required short absorption recovery time of $5 \mathrm{ps}$ is obtained by heavy ion irradiation [6].

The SA reflectivity increases with the incident power, so that low power levels are significantly attenuated while high power levels are much less attenuated. This results in a signal extinction ratio enhancement, which is called the contrast.

After reflection at the SA, the signal propagates through an SOA, which possesses a saturating gain function. The signal saturates the SOA gain, so that high power pulses are less amplified than low power pulses. This tends to keep the SOA output power constant and to limit power fluctuations. The SOA is acting as a limiting amplifier, which results in an equalization of the "mark" levels. Thus, the transmission functions of the SA and the SOA are complementary and if both are employed, then full $2 \mathrm{R}$ regeneration is ensured.

A holding beam is coupled to the signal at the SOA input to reduce the gain recombination time so as to be $10-\mathrm{Gb} / \mathrm{s}$ compatible [7]. Under these conditions no significant patterning effects are observed. An optical filter (OF) prevents wideband ASE accumulation. 


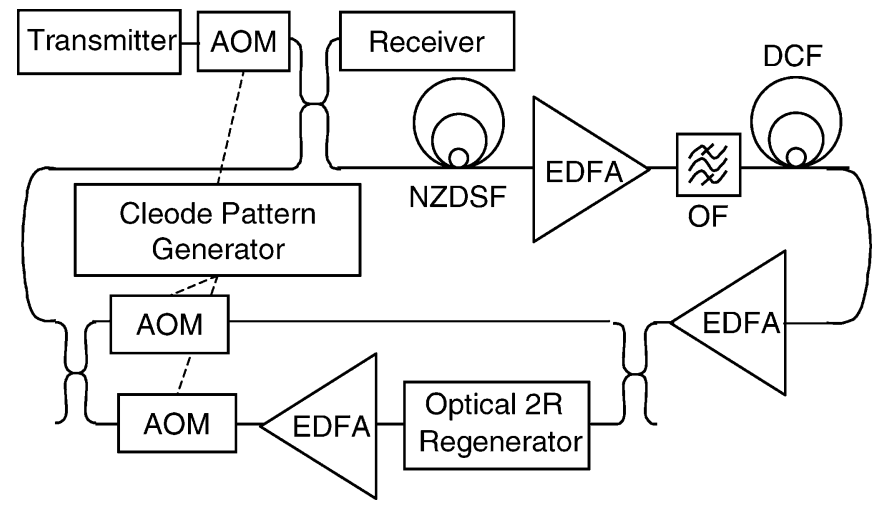

Fig. 2. Two-path recirculating loop.

\section{TRANSMISSION EXPERIMENT}

The 10-Gb/s transmission experiment is carried out with a 100-km-long two-path recirculating loop, emulating regeneration spans longer than the available fiber length.

Nonzero dispersion-shifted fiber with a chromatic dispersion of $4 \mathrm{ps} / \mathrm{nm} / \mathrm{km}$ is used in the loop. The fiber link dispersion is partially compensated with dispersion-compensating fiber. Fig. 2 shows the experimental setup of the recirculating loop.

Span input power is set to $+2 \mathrm{dBm}$. Amplification is ensured by two erbium-doped fiber amplifiers. OFs prevent ASE peak accumulation. The transmitter consists of a $2^{15}-1$ pseudorandom bit sequence combined with a logical gate which produces a return-to-zero electrical signal. A receiver problem prevented operation at $2^{31}-1$; however, the device should be capable of working at this sequence length as some experiments with the same type of SA have been reported with this sequence length [2]. This signal modulates an optical source through a $\mathrm{LiNbO}_{3}$ modulator which produces 50 -ps pulses.

After propagation through the transmission fiber, the signal can be switched through either the nonregenerated path or through the regenerated path, using $3-\mathrm{dB}$ couplers and acoustooptic modulators driven by a commercial pattern generator (from CLEODE). Switching between the paths in this way allows the span between two regenerations be varied.

\section{RESULTS}

First, the impact of the regeneration span was studied at a signal wavelength of $1557 \mathrm{~nm}$. Fig. 3 presents the bit-error-rate (BER) evolution as a function of the distance without regenerator (full triangles) and with regenerators (open symbols) located at 1 (triangles), 2 (circles), 3 (squares), and 6 (crosses) laps. With regeneration at each lap, 140 cascades can be achieved with a BER of $10^{-8}$. Best propagation distance is obtained with a regeneration spacing of $200 \mathrm{~km}$ where a distance of $20000 \mathrm{~km}$ can be achieved, with a BER of $10^{-8}$.

However, we show that a larger spacing is still quite relevant, since, with regeneration every $600 \mathrm{~km}$, the distance is still doubled $(4000 \mathrm{~km})$ for a BER of $10^{-8}$ with only six regenerators cascaded. And finally, the performance is more than tripled for a BER of $10^{-4}$ (if forward-error correction (FEC) is considered), allowing a transmission over $11000 \mathrm{~km}$ with only 18 regenerators.

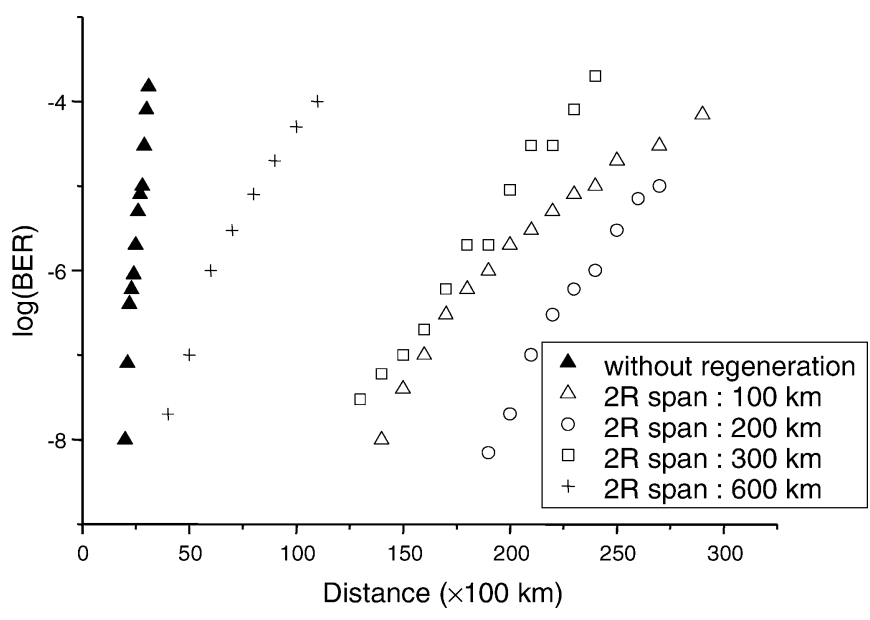

Fig. 3. BER evolution versus distance without and with different regeneration spans.

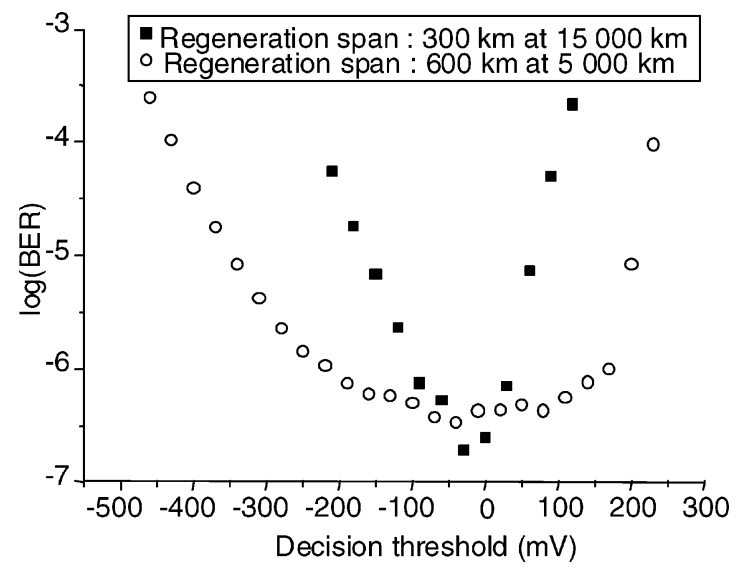

Fig. 4. BER evolution versus decision threshold (millivolts) for different regeneration spans and distances.

It may seem surprising that best performance is not obtained for the shortest regeneration span. However, in a $2 \mathrm{R}$ regeneration link, both amplitude noise and timing jitter limit the transmission.

In order to show the impact of timing jitter, we studied the BER evolution as a function of the decision threshold of the receiver. In [8, Fig. 5], we showed numerically that the power probability density function transformation through a reshaping gate leads to a plateau in the evolution of the BER as a function of the decision threshold of the receiver. In [9], we showed experimentally, using an optical 3R regenerator, that the plateau width keeps on broadening as the number of laps increases when amplitude noise limits the transmission (timing jitter is negligible in a $3 \mathrm{R}$ case). In the present experiment, we study the BER evolution at two different $2 \mathrm{R}$ regeneration spans. For each regeneration span the distance was chosen to have an easily measurable BER (between $5 \cdot 10^{-6}$ and $10^{-7}$ ). Fig. 4 shows the evolution of the BER as a function of the decision threshold corresponding to a distance of $15000 \mathrm{~km}$ for a $300-\mathrm{km}$ span (squares) and a distance of $5000 \mathrm{~km}$ for a $600-\mathrm{km}$ span (circles). In the case of a $600-\mathrm{km}$ span, a plateau appears, which is indicative of operation in the regeneration regime [8]. So we might, therefore, expect that in the case of $300 \mathrm{~km}$, a plateau would also 


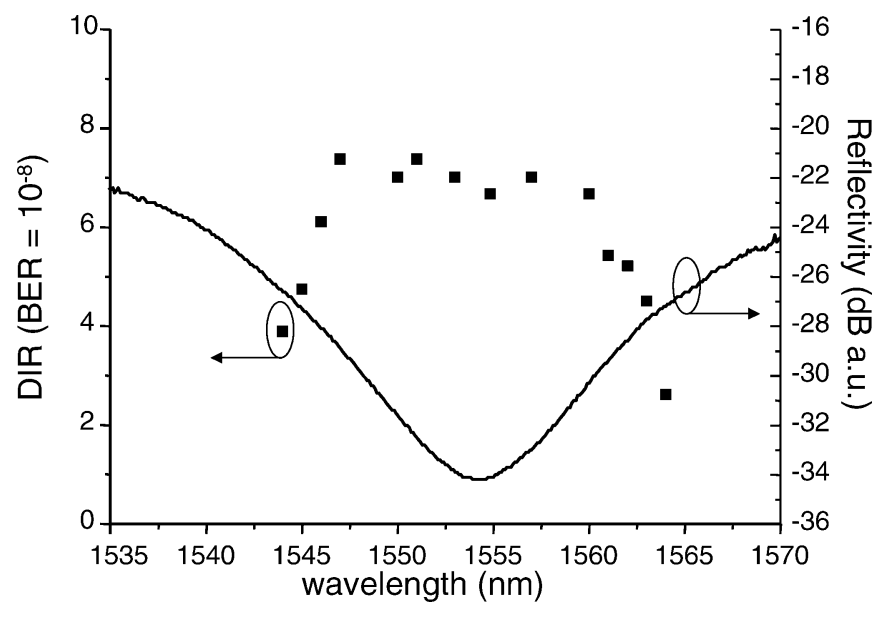

Fig. 5. DIR (at BER $=10^{-8}$ ) evolution and small signal reflectivity versus signal wavelength for a regeneration span of $100 \mathrm{~km}$.

appear as amplitude noise reduction is better when the regeneration span decreases. This is, however, not what is observed. The disappearance of the plateau in the case of a $300-\mathrm{km}$ span is due to timing jitter accumulation, as we now explain.

For a given distance, the longer the regeneration span is indeed, the more amplitude noise accumulates; but the shorter the regeneration span is, the more timing jitter accumulates. Therefore, in a $2 \mathrm{R}$ regenerated link, there is a best compromise between timing jitter accumulation and amplitude noise accumulation which is not obtained with the shortest regeneration span. In our experiment, the best compromise is obtained for a $200-\mathrm{km}$ span as already observed in Fig. 3.

We have also investigated the regeneration behavior with respect to the signal wavelength. We define the distance improvement ratio (DIR) as the ratio of distances covered with regeneration and without regeneration for a given BER. Fig. 5 presents the evolution of the DIR with signal wavelength for a BER of $10^{-8}$ and a regeneration span of $100 \mathrm{~km}$ (square symbols). Results show that the DIR is better than 6.5 over more than $13 \mathrm{~nm}$ (from 1547 to $1560 \mathrm{~nm}$ ), demonstrating the wideband behavior of the device.

On the right-hand axis of this graph, we plot the small signal reflectivity of the SA. The highest contrast is obtained at the resonance wavelength; so we might expect that the performance curve would have the same shape than the small signal spectrum curve but inverted, i.e., the performance would decrease with the contrast. The result obtained here tends to show that the performance does not increase with the contrast, but that there is a minimum contrast for the device to be efficient. A higher contrast does not improve the performance.

\section{CONCLUSION}

We have reported on a simple and compact $2 \mathrm{R}$ regenerator based on an SA in a vertical microcavity and an SOA. This device is fully compatible with photonic integration, which could allow compact and low cost WDM $2 \mathrm{R}$ regeneration.

We have also studied, for the first time, the influence of the regeneration span on the DIR when regenerators are cascaded, thanks to our two-path recirculating loop.

The most relevant performance, from a cost point of view, may be that for a regeneration spacing of $600 \mathrm{~km}$, which allows transmission over $11000 \mathrm{~km}$ with a BER of $10^{-4}$ without FEC, only 18 regenerators are needed.

It was also shown that the device operates over a spectral range as wide as $13 \mathrm{~nm}$.

\section{ACKNOWLEDGMENT}

The authors thank all the partners of the project ASTERIX who have contributed to the fabrication of the saturable absorber component, and especially J. Decobert, J. Landreau, O. Le Gouezigou, F. Poingt, L. Le Gouezigou, and F. Pommereau from Alcatel Thales III-V Laboratory, and J. Mangeney and S. Sauvage from the Institut d'Electronique Fondamentale (IEF-CNRS UMR 8622). They are also grateful to $\mathrm{H}$. Bernas for the component irradiation in the Aramis accelerator of the CSNSM-CNRS in Orsay.

\section{REFERENCES}

[1] A. Shen et al., "4-channel saturable absorber module for high bit-rate regenerated WDM transmission," presented at the ECOC 2002, Copenhagen, Denmark, Paper Tu 5.4.5.

[2] D. Rouvillain et al., "Optical 2R regenerator based on passive saturable absorber at $40 \mathrm{Gbit} / \mathrm{s}$ for WDM long haul transmissions," Electron. Lett., vol. 38, no. 19, pp. 1113-1114, 2002.

[3] M. Gicquel-Guezo et al., "290 fs switching time of Fe-doped quantum well saturable absorbers in a microcavity in $1.55 \mu \mathrm{m}$ range," Appl. Phys. Lett., vol. 85, no. 24, pp. 5926-5929, 2004.

[4] Z. Bakonyi et al., "In-line saturable absorber in transmission systems with cascaded semiconductor optical amplifiers," IEEE Photon. Technol. Lett., vol. 12, no. 5, pp. 570-572, May 2000.

[5] D. Massoubre et al., "Low switching energy saturable absorber device for $40 \mathrm{Gbit} / \mathrm{s}$ networks," in Integrated Photonics Research. San Francisco, CA: OSA, 2004.

[6] J. Mangeney et al., "Ultrafast saturable absorption at $1.55 \mu \mathrm{m}$ in heavy-ion irradiated quantum well vertical cavity," Appl. Phys. Lett., vol. 76, no. 11, pp. 1371-1373, 2000.

[7] R. J. Manning, D. A. O. Davies, and J. K. Lucek, "Recovery rates in semiconductor laser amplifiers: Optical and electrical bias dependencies," Electron. Lett., vol. 30, no. 15, pp. 1233-1234, 1994.

[8] J. C. Simon et al., "All-optical regeneration techniques," Annales Telecommun., vol. 58, no. 11-12, pp. 1708-1724, 2003.

[9] M. Gay et al., "Experimental study of reshaping retiming gates for 3R regeneration," in Optical Networks and Technologies Conf. OpNeTec Pisa, 2004, pp. 545-551. 